\title{
Efecto de la congelación y los periodos de almacenamiento en la calidad organoléptica de la carne y sus compuestos aromáticos
}

\author{
M. Bueno ${ }^{1}$, M.M. Campo ${ }^{2}$, J. Cacho ${ }^{1}$, V. Ferreira ${ }^{1}$, A. Escudero ${ }^{1}$ \\ ${ }^{1}$ Laboratorio de Análisis de Aroma y Enología (LAAE) \\ Instituto de Investigación en Ingeniería de Aragón (I3A) \\ Universidad de Zaragoza, Dpto. Química Analítica, c/ Pedro Cerbuna 12, 5009, Zaragoza, Spain. \\ Tel.+34-976761000 (Ext. 3328), Fax+34-976761292, e-mail: mobueno@unizar.es \\ ${ }^{2}$ Departamento de Producción Animal y Ciencia de los Alimentos \\ Universidad de Zaragoza \\ c/ Miguel Servet, 117, 50013 Zaragoza, Spain
}

\begin{abstract}
Una realidad del mercado de la carne de ovino es la estacionalidad en el abastecimiento de corderos, lo que implica fluctuaciones en el precio. Se ha estudiado el efecto que tiene la congelación en la calidad organoléptica de la carne de ovino, con especial interés en la percepción del aroma, para intentar estabilizar este mercado. Se han comparado 7 tratamientos distintos: tres métodos de congelación (túnel de congelación, cámara de aire dinámica y cámara de aire estática) durante dos periodos de almacenamiento (1 y 10 meses), y se han comparado con carne fresca. El análisis sensorial solamente mostró diferencias significativas ( $p=95 \%)$ en 2 de los 11 parámetros estudiados, jugosidady flavor a cordero. Por otro lado, si se analizan los compuestos volátiles aromáticos del cordero con GC-MS o GC-GC-MS, provenientes de extractos obtenidos usando la metodología de extracción en grill descrita por Bueno et al. (2011), se observan diferencias significativas $(p=95 \%)$ para muchos compuestos carbonílicos. Este hecho implica que se generan en diferente proprción según el tratamiento. Sin embargo, no hay diferencias significativas en los niveles relativos de los compuestos. Esto significa que independientemente del tratamiento la proporción de los aromas de oxidación es la misma, y podría ser una de las razones por las cuales no se han encontrado diferencias en el análisis sensorial para notas diferentes al flavor a cordero. También existen compuestos como el furaneol, el ácido octanoico y el 2-fenoxietanol, que destacan como indicadores de congelación.
\end{abstract}

